\title{
Coronary anomaly: anomalous right coronary artery originates from the left sinus of Valsalva and coursing between the pulmonary artery and aorta
}

\author{
This article was published in the following Dove Press journal: \\ Clinical Interventions in Aging \\ 10 September 2013 \\ Number of times this article has been viewed
}

\author{
Xuguang Qin' \\ Weiguo Xiong' \\ Enben Guan² \\ Chunpeng $\mathrm{Lu}^{\prime}$ \\ 'Department of Cardiology, First \\ Affiliated Hospital of Tsinghua \\ University, Beijing, People's Republic \\ of China; ${ }^{2}$ Department of Pediatric, \\ The Affiliated Hospital of Qingdao \\ Medical College, Qingdao, People's \\ Republic of China
}

\begin{abstract}
Coronary artery anomalies (CAAs) are present at birth, but are usually asymptomatic and are found during coronary angiography or multi-slice computed tomography (MSCT) detection. The most common coronary anomaly is the separating origin of left anterior descending coronary artery (LAD) and left circumflex artery (LCX) from the left sinus of Valsalva, and this variant is benign. Herein, we present three extremely rare cases of anomalous right coronary artery (RCA) detected incidentally during routine coronary angiography and confirmed by multi-slice computed tomography (MSCT) technique. All the anomalous right coronary artery coursed between the pulmonary artery and aorta. We discuss how to make an accurate diagnosis for appropriate management.
\end{abstract}

Keywords: coronary anomaly, anomalous right coronary artery, coronary angiography, elderly

\section{Introduction}

Coronary artery anomalies (CAAs) are present at birth, but are usually asymptomatic and are found during coronary angiography or multi-slice computed tomography (MSCT) detection. Their prevalence is less than $1.3 \%$ based on a published series. ${ }^{1-4}$ The most common coronary anomaly is the separating origin of the left anterior descending coronary artery (LAD) and left circumflex coronary artery (LCX) from the left sinus of Valsalva. The second most common anomaly is origination of the LCX artery from the right coronary artery (RCA) or right sinus of Valsalva. Herein, we present three extremely rare cases of anomalous right coronary artery (RCA) detected incidentally during routine coronary angiography. The anomalous RCA originates from the left sinus of Valsalva, and a 64-slice MSCT detection of the heart was performed to distinguish the origin and course of the anomalous RCA. The results showed that the anomalous right coronary artery originated from the left sinus of Valsalva and coursing between the pulmonary artery and aorta; these are extremely rare. We discuss how to make an accurate diagnosis for appropriate management.

\section{Case one}

A 64-year-old woman complained of gradually progressive exertional angina pectoris for 3 months. Electrocardiogram was normal. Coronary angiogram was performed through a trans-radial approach. Coronary angiogram demonstrated the left main coronary artery (LMCA) and LAD were normal, but there was mild stenosis in the middle of the LCX 
segment (Figure 1A). Repeated attempts to cannulate the RCA were not successful (Figure 1B). A nonselective injection into the left coronary sinus with a pigtail catheter demonstrated an anomalous RCA arising from the left sinus. In order to confirm the anomalous origin and course of RCA, a 64-slice MSCT detection of the heart was performed on a 64-slice machine (Philips 64 Slice, Philips, Amsterdam, The Netherlands). The results showed the anomalous RCA originating from the left sinus of Valsalva, anteriorly, then taking an acute bend to course between the aorta and pulmonary artery trunk. The anomalous RCA was classified as type L-II B subtype (Figure $1 \mathrm{C}$ and $\mathrm{D}$ ). The patient was treated medically.

\section{Case two}

A 60-year-old woman presented with dyspnea after physical exertion for more than 2 months. Electrocardiogram showed ST depression in inferior leads II, III, aVF. Coronary angiogram was performed through trans-femoral approach. Coronary angiogram demonstrated the LMCA was normal, there was borderline narrowing in the proximal segment of LAD, and the LCX was small and patent. Repeated attempts to cannulate the RCA were successful and the result showed that the anomalous RCA originated from the left sinus of Valsalva and there was severe narrowing in the posterior descending
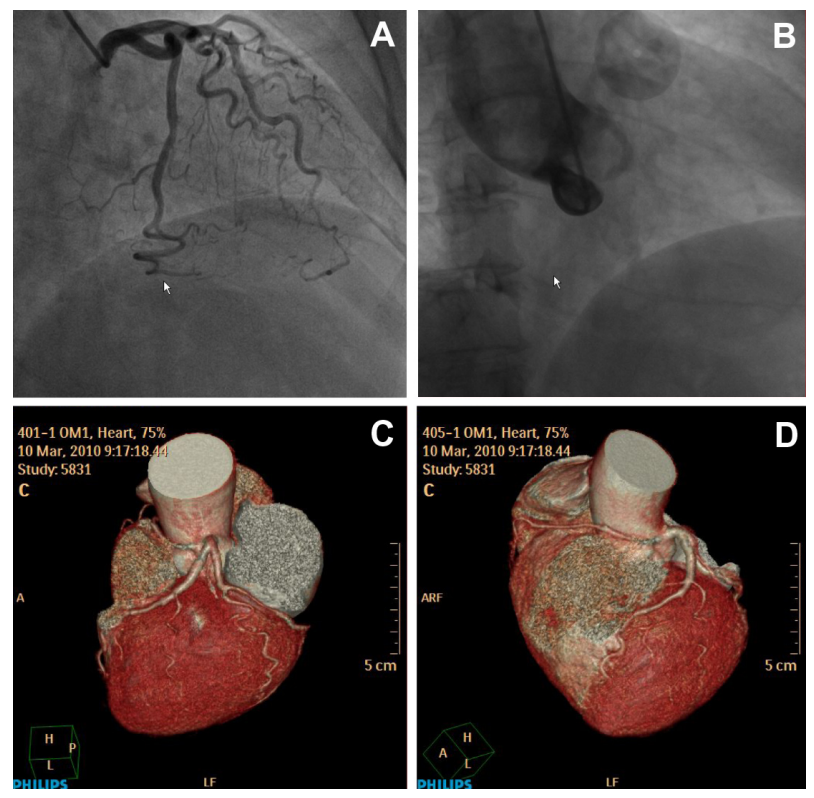

Figure I (A) RAO caudal view shows a mild lesion in the middle of the LCX artery and (B) LAO view demonstrates no RCA in the right sinus of Valsalva. (C) Reconstructed three-dimensional image obtained by the volume-rendering technique shows the anomalous RCA with an interarterial course. (D) Similarly, the anomalous RCA between the aorta and pulmonary trunk can be seen.

Abbreviations: LAO, left anterior oblique; LCX, left circumflex coronary artery; $\mathrm{RAO}$, right anterior oblique; RCA, right coronary artery. coronary artery (PDA). Reconstructed three-dimensional images were performed to verify the course of the anomalous RCA. The results showed the anomalous RCA originating from the left sinus anterior to the left coronary artery ostium, then taking an acute bend to course between the aorta and pulmonary artery. The right coronary artery anomaly was classified as type L-II B subtype (Figure 2A and B). Neither percutaneous coronary intervention $(\mathrm{PCI})$ nor coronary artery bypass grafting $(\mathrm{CABG})$ was performed, and the patient was only treated medically.

\section{Case three}

A 66-year-old man presented with an acute anterior myocardial infarction and had reperfusion therapy with thrombolytic agent. Electrocardiograms showed ST segment elevation in precordial leads $\mathrm{V}_{1-3}$ with normal creatininase values and troponin $\mathrm{T}$ concentration of $0.02 \mu \mathrm{g} / \mathrm{L}$. The patient underwent cardiac catheterization because of post-infarct unstable angina. Angiography demonstrated that the right coronary artery and left main coronary arteries were originating from the same ostium of the left sinus (Figure 3A). The left main stem was patent, the LAD artery had $90 \%$ stenosis in the proximal segment, and the LCX artery was patent and non-dominant. The anomalous RCA had a high-grade $90 \%$ stenosis in the proximal (Figure 3B). A 6-F left Amplatz 1.5 guiding catheter was used to engage the left coronary system to perform PCI to the anomalous RCA and LAD, one stent was implanted at the proximal of the RCA segment and another stent deployed in the proximal of the LAD segment. The final angiographic result was excellent (Figure 3C). In order to confirm the origin and course of anomalous RCA, a 64-slice MSCT detection of the heart was performed on a 64-slice machine (Philips). The results showed the

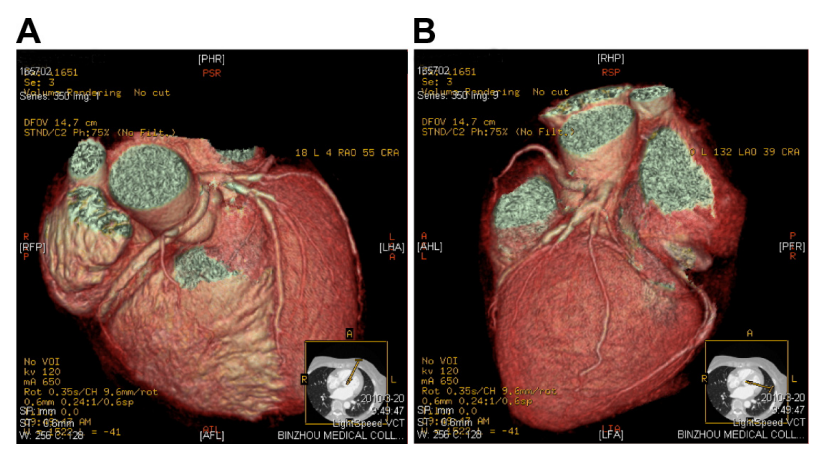

Figure 2 (A) Reconstructed three-dimensional image obtained using the volumerendering technique shows the anomalous RCA with an interarterial course. (B) The origin of the anomalous RCA is evident.

Abbreviation: RCA, right coronary artery. 

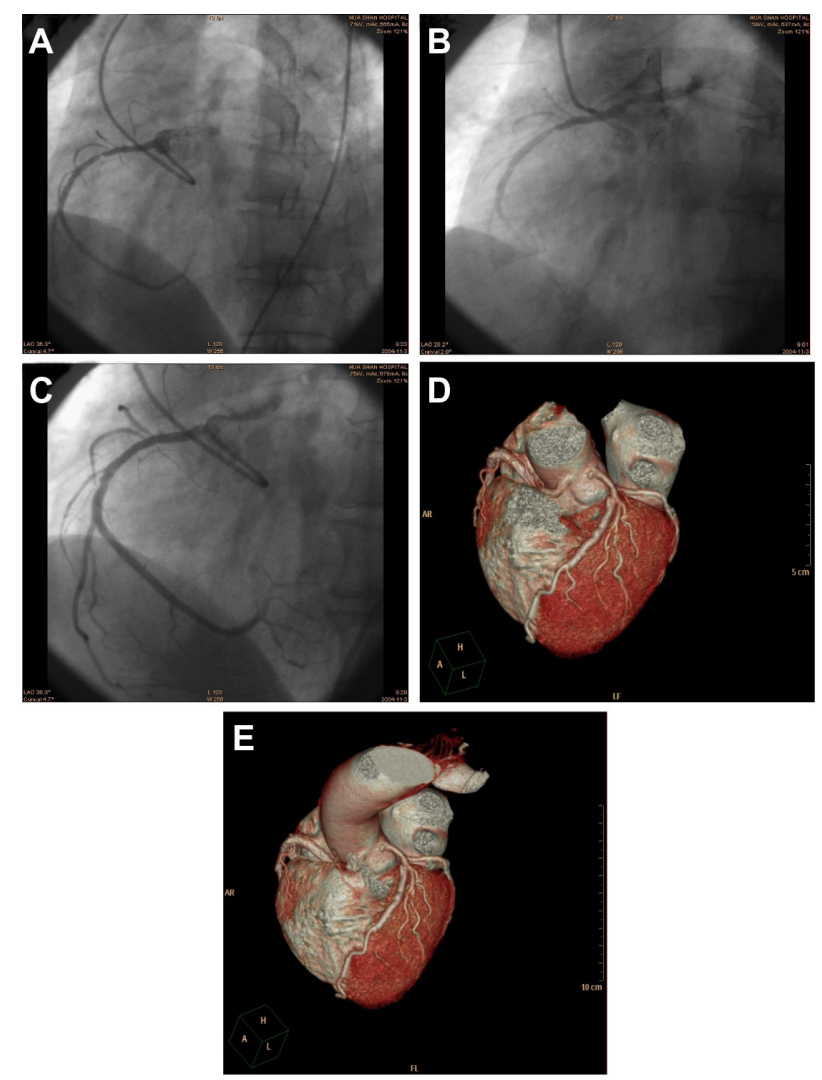

Figure 3 (A) LAO cranial view shows an anomalous RCA arising from the left sinus of Valsalva. (B) LAO cranial view shows a high-grade $90 \%$ stenosis in the proximal RCA. (C) LAO cranial view shows a final good angiographic result after $\mathrm{PCl}$ procedure. (D) Reconstructed three-dimensional image obtained by the volume-rendering technique shows the anomalous RCA with an interarterial course. (E) The anomalous RCA originates from the left sinus of Valsalva.

Abbreviations: $\mathrm{LAO}$, left anterior oblique; $\mathrm{PCl}$, percutaneous coronary intervention; $\mathrm{RCA}$, right coronary artery.

anomalous RCA originating from the left sinus of Valsalva, anteriorly, then taking an acute bend to course between the aorta and pulmonary artery trunk. The anomalous coronary artery was classified as type L-II B subtype.

\section{Discussion}

CAAs are present at birth, but are usually asymptomatic and are found during coronary angiography or MSCT detection. Their prevalence is less than $1.3 \%$ based on a published series. ${ }^{1-4}$ The most common coronary anomaly is the separating origin of the LAD and LCX from the left sinus of Valsalva. The second most common anomaly is the origination of the LCX artery from the RCA or right sinus of Valsalva. Anomalous right coronary artery deriving from the left coronary sinus of Valsalva is rare; its prevalence is about $0.17 \% 0^{1,2}$ The three cases we reported herein all have anomalous RCA originating from the left sinus of Valsalva. The anomalous RCA arises from an orifice located anterior to the left main ostium in the left sinus of Valsalva and may course between the aorta and pulmonary artery before reaching the right atrioventricular (AV) groove (Figure 1D). This anomaly is suspected when the RCA ostium is unable to be located in the right sinus of Valsalva and collateral vessels are absent. The ectopic RCA is difficult to cannulate because of its slit-like orifice and odd angulation. Ostial occlusion due to aortic expansion during exercise may result in myocardial ischemia. ${ }^{2}$ Clinical sequelae are rare, although angina pectoris, myocardial infarction, ventricular tachycardia, syncope, and sudden cardiac death in the absence of coronary atherosclerosis have been reported. ${ }^{3,4}$

In order to confirm the anomalous origin and course of the anomalous RCA, a MSCT detection of the heart was performed using a 64-slice machine (Philips). The results showed the anomalous RCA originating from the left sinus, posteriorly, then taking an acute bend to course between the aorta and pulmonary artery. There are four subtypes based on the anatomic course of the aortic artery: the aberrant vessel may course posterior to the aorta (retroaorta), or between the ascending aorta and pulmonary artery (interarterial), prepulmonary artery (prepulmonic), or septal (subpulmonic). ${ }^{3,6,7}$ Although the interarterial subtype is extremely rare, this subtype of patient may present with symptoms of angina pectoris, myocardial infarction, ventricular tachycardia, syncope, and sudden cardiac death. Currently, these patients are submitted to cardiac surgery treatment, according to 2008 American College of Cardiology (ACC)/American Heart Association (AHA) guidelines for the management of adults with congenital heart disease. ${ }^{5}$ The three cases of anomalous RCA we reported were classified as type L-II B subtype (Figures 1C, 2A, 2B and 3D and E) and finally results were confirmed by 64-slice computed tomography of the heart. The first two cases were treated with oral medications, while on the third one PCI was performed; all patients are free of ischemic symptoms during follow-up.

We present these cases in an attempt to highlight their significance. These anomalies are considered to be an independent risk factor for adverse cardiovascular events including sudden cardiac death and dealing with these lesions is a challenge to cardiologists or cardiac surgeons.

\section{Conclusion}

We report three extremely rare cases of anomalous RCA that originates from the left sinus of Valsalva, and it courses between the pulmonary artery and aorta. These coronary anomalies are considered to be independent risk factors for 
adverse cardiovascular events, so we present these cases in an attempt to highlight their significance.

\section{Disclosure}

The authors report no conflicts of interest in this work.

\section{References}

1. Yamanaka O, Hobbs RE. Coronary artery anomalies in 126,595 patients undergoing coronary arteriography. Cathet Cardiovasc Diagn. 1990;21(1):28-40.

2. Roberts WC, Siegel RJ, Zipes DP. Origin of the right coronary artery from the left sinus of valsalva and its functional consequences: analysis of 10 necropsy patients. Am J Cardiol. 1982;49(4):863-868.

3. Barth CW, Bray M, Roberts WC. Sudden death in infancy associated with origin of both left main and right coronary arteries from a common ostium above the left sinus of Valsalva. Am J Cardiol. 1986;57(4):365-366.

4. Taylor AJ, Rogan KM, Virmani R. Sudden cardiac death associated with isolated congenital coronary artery anomalies. J Am Coll Cardiol. 1992;20(3):640-647.
5. Warnes CA, Williams RG, Bashore TM, et al; American College of Cardiology; American Heart Association Task Force on Practice Guidelines (Writing Committee to Develop Guidelines on the Management of Adults With Congenital Heart Disease); American Society of Echocardiography; Heart Rhythm Society; International Society for Adult Congenital Heart Disease; Society for Cardiovascular Angiography and Interventions; Society of Thoracic Surgeons. ACC/AHA 2008 guidelines for the management of adults with congenital heart disease: a report of the American College of Cardiology/American Heart Association Task Force on Practice Guidelines (Writing Committee to Develop Guidelines on the Management of Adults With Congenital Heart Disease). Developed in Collaboration With the American Society of Echocardiography, Heart Rhythm Society, International Society for Adult Congenital Heart Disease, Society for Cardiovascular Angiography and Interventions, and Society of Thoracic Surgeons. J Am Coll Cardiol. 2008;52(23):e143-e263.

6. Angelini P, Velasco JA, Flamm S. Coronary anomalies: incidence, pathophysiology, and clinical relevance. Circulation. 2002;105(20): $2449-2454$.

7. Angelini P. Coronary artery anomalies: an entity in search of an identity. Circulation. 2007;115(10):1296-1305.
Clinical Interventions in Aging

\section{Publish your work in this journal}

Clinical Interventions in Aging is an international, peer-reviewed journal focusing on evidence-based reports on the value or lack thereof of treatments intended to prevent or delay the onset of maladaptive correlates of aging in human beings. This journal is indexed on PubMed Central, MedLine, the American Chemical Society's 'Chemical Abstracts

\section{Dovepress}

Service' (CAS), Scopus and the Elsevier Bibliographic databases. The manuscript management system is completely online and includes a very quick and fair peer-review system, which is all easy to use. Visit $\mathrm{http}: / /$ www.dovepress.com/testimonials.php to read real quotes from published authors. 